\title{
Survival rate and neurodevelopmental outcome of extremely premature babies: an 8-year experience of an Italian single neonatal tertiary care center
}

\author{
Sara Uccella, Agnese De Carli, Ida Sirgiovanni, Paola Schiavolin, Giuseppe Damiano, \\ Beatrice Ghirardi, Francesca Maglioli Carpano, Laura Bassi, Silvana Gangi, \\ Odoardo Picciolini, Monica Fumagalli, Fabio Mosca \\ Department of Clinical Sciences and Community Health, Fondazione IRCCS Ca' Granda, Università \\ degli Studi di Milano, Milan, Italy
}

\begin{abstract}
Extremely preterm babies are at major risk for adverse neurodevelopmental outcome, being the gestational age (GA) the main determinant for a good-quality survival. Aim of this retrospective study was to investigate the neurodevelopmental outcome in a population of extremely preterm babies admitted to a single neonatal tertiary care unit over an 8 -year period. All babies born between $23^{+0}$ and $25^{+6}$ weeks of GA from January 2003 until December 2010 were retrospectively enrolled. Perinatal and neonatal variables were recorded. Motor and cognitive development was assessed using the neurofunctional scale (NFS) and the Griffith's scales at 2 years. Fifty-five out of 122 infants survived to discharge. Survival rates doubled for each additional gestational week from 23 to $25: 16 \%, 38 \%$ and $74 \%$ at 23,24 and 25 weeks GA respectively. Forty-six infants were evaluated at 2 years. A poor cognitive and motor outcome was observed in all babies born at 23 weeks. Griffith's general quotient (GQ) was $\geq 76$ in $62 \%$ and $\geq 88$ in $33 \%$ of babies born between 24 and 25 weeks. No severe motor disabilities were found in $81 \%$ of babies born between 24 and 25 weeks. Preterm premature rupture of membranes, absence of prenatal steroids, intrauterine growth restriction, male, lower GA and major brain abnormalities at magnetic resonance imaging (MRI) were significantly associated with worse NFS and lower mean GQ at 2 years of age. GA, gender and abnormal MRI findings remained significantly associated with impaired NFS at the multivariate analysis. Survival rates and neurodevelopmental outcome improved with each week of
\end{abstract}

Correspondence: Monica Fumagalli, Department of Clinical Sciences and Community Health, Fondazione IRCCS Ca' Granda, Università degli Studi di Milano, via Commenda 12, 20121 Milan, Italy.

Tel: +39.02.55032951 - Fax: +39.02.55032217.

E-mail: monica.fumagalli@mangiagalli.it

Key words: Preterm; Neurodevelopment; Survival; Neurofunctional; MRI.

Received for publication: 4 March 2015.

Accepted for publication: 31 July 2015.

This work is licensed under a Creative Commons Attribution NonCommercial 3.0 License (CC BY-NC 3.0).

(O)Copyright S. Uccella et al., 2015

Licensee PAGEPress, Italy

La Pediatria Medica e Chirurgica 2015; 37:106

doi:10.4081/pmc.2015.106
GA. These results are relevant for clinicians counselling families facing an unavoidable extremely preterm birth.

\section{Introduction}

Preterm birth is a major paediatric public health problem as prematurity is associated with a considerable risk to develop cognitive, behavioral, neurosensory, and motor disabilities. The rates of preterm birth have risen up in many European countries, ${ }^{1}$ and a concomitant successful improvement in survival has been reported through the years. ${ }^{2}$ However, a missing reduction in the prevalence of severe disability still characterizes the extremely prematurely-born population, ${ }^{2}$ being the gestational age the major discriminant for a good-quality survival of these extremely preterm babies:2-4 the lower the gestational age, the higher the risk of death and neurodevelopmental impairment.

Brain development after extremely preterm birth is complex and poorly understood ${ }^{5}$ and the outcome in the very early preterm infants depends on a mixture of well-described haemorrhagic or ischemic injuries together with less known maturational and trophic disturbances affecting the developing brain. ${ }^{6} \mathrm{~A}$ range of perinatal factors, such as mother's diseases and habits - e.g. nutrition, ${ }^{7}$ alcohol abuse ${ }^{8}$ etc. - infection and inflammation, $, 3,9$ fetal growth restriction ${ }^{10,11}$ as well as the hospital course and exposure to systemic illness - chronic lung disease, postnatal infections ${ }^{12-15}$ - have been shown to further exacerbate disturbances in brain maturation with a negative impact on neurodevelopmental outcome.

Recently, the EPICure study reported short term outcomes after extreme preterm birth ( $\leq 26$ weeks gestation) in England, comparing two birth cohorts in 1995 and 2006: a higher survival rate has been observed. ${ }^{4}$ Although an overall increase in severe neurological impairment was shown, a bigger proportion of babies born at 24 and 25 weeks' gestation survived. ${ }^{2}$

Aim of this retrospective study was to investigate the neurodevelopmental outcome and related perinatal risk factors in a population of extremely preterm babies ( $23+0$ and $25+6$ weeks of gestation) admitted to a single neonatal tertiary care unit over an 8-year period.

\section{Materials and Methods}

We retrospectively collected data on all extremely preterm babies with GA at birth between $23+0$ to $25+6$ admitted to the neonatal tertiary care center (intensive care unit) Department of Clinical Sciences 
and Community Health, Fondazione IRCCS Ca' Granda, Università degli Studi di Milano, Milan, Italy, over the course of 8 years (since January 2003 till December 2010).

Prenatal and perinatal characteristics were recorded: spontaneous or assisted reproduction, multiple pregnancy, mode of delivery, prenatal steroids, obstetric complications as gestational hypertension, severe intrauterine growth restriction (IUGR), preterm premature rupture of membranes (PPROM), maternal fever $>38^{\circ} \mathrm{C}$.

The following neonatal variables and main complications during the neonatal period were collected: gender, GA based on the last menstrual period and first-trimester ultrasonogram, birth weight, singletons or twins, Apgar score at 5 minutes, pneumothorax, patent ductus arteriosus (PDA) requiring any treatment, bronchopulmonary dysplasia (BPD) defined as oxygen dependency up to 36 weeks corrected age, rethinopathy of prematurity (ROP) requiring any treatment, abdominal surgery, any congenital malformation.

Infants with birth weight $>10^{\text {th }}$ or $\leq 10^{\text {th }}$ percentile for $\mathrm{GA}$, according to the North-Italian growth charts, were classified as appropriate for gestational age (AGA) or small for gestational age (SGA) respectively. ${ }^{16}$ All babies underwent brain magnetic resonance imaging (MRI) at term corrected age (TCA). MR images were classified as normal, with minor abnormalities and severely abnormal (cystic periventricular leukomalacia, arterial or venous stroke, multiple white matter punctate lesions, post-hemorrhagic ventricular dilatation). Infants were followed-up by assessments of neurodevelopment at two years of corrected age. The neurofunctional scale (NFS) ${ }^{17}$ and the Griffith's Developmental Scales II (GMDS II) ${ }^{18}$ were used. The neurofunctional scale was assigned as follows: 0 , normal function; 1 , mild impairment of function (no limitations); 2, moderate impairment of function (possible but limited); 3 , severe impairment of function (possible only with the use of facilitators or assisted devices); 4, function not possible. The Griffith's scales consist of six sub-scales (locomotor, personal-social, hearing-speech, eye-hand coordination, performance and logical scales) which are combined to give the total score, the general quotient (GQ). The Griffith's GQ was defined as: normal $\geq 88$, mild cognitive delay between 76 and 87 , severe cognitive deficit $<76$. Severe disability was defined as either NFS score $\geq 3$ or $\mathrm{GQ}<76$.

Analysis of variance and multivariate analysis were performed to evaluate the effect of tested factors on outcomes.

\section{Results}

One hundred twenty-two infants were born during the study period; 55 babies (45\%) survived to discharge but 9 were lost at follow-up. Twenty-six out of the 46 babies followed-up (56\%) were born at 25 weeks' gestation, 16 (35\%) at 24 and $4(9 \%)$ at 23 .

The survival rates according to gestational age are shown in Table 1: survival rate doubled for each additional gestational week from 23 to 25 but no significant differences were observed between two study periods (period 1: 2003-2006; period 2: 2007-2010). Prenatal, perinatal and neonatal variables are shown in Tables 2 and 3 .

Forty-six infants were evaluated by both neurofunctional assessment and Griffith's scale at 2 years CA; results are reported in Figure 1. The neurodevelopmental outcome clearly differed according to gestational age at birth: none of the children born at 23 weeks had a normal neurofunctional or neurobehavioral assessment.

At 2 years of age $34 / 46$ babies ( $74 \%$ ) had a NFS score $<3$, without any significant change between the 2 study periods (77\% in period 1 versus $72 \%$ in period 2). Ten to sixteen (62\%) babies born at 24 weeks and $24 / 26$ (92\%) born at 25 weeks had NFS score $<3$. A Griffith's GQ $\geq 76$ was observed in 26/46 (56\%) babies: 7/16 (44\%) born at 24 and 19/26 (73\%) born at 25 weeks.
Table 1. Survival rate.

\begin{tabular}{lccc} 
& $2003-2010$ & $2003-2006$ & $2007-2010$ \\
& $(\%)$ & $(\%)$ & $(\%)$ \\
All weeks & $55 / 122(45)$ & $20 / 46(43)$ & $35 / 76(46)$ \\
23 weeks & $5 / 32(16)$ & $2 / 16(13)$ & $3 / 16(19)$ \\
\hline 24 weeks & $18 / 47(38)$ & $6 / 14(43)$ & $12 / 33(37)$ \\
25 weeks & $32 / 43(74)$ & $12 / 16(75)$ & $20 / 27(74)$ \\
\hline
\end{tabular}

Table 2. Prenatal and perinatal factors.

\begin{tabular}{lc} 
& N $(\%)$ \\
Assisted reproduction & $9 / 46(20)$ \\
Gestational hypertension & $8 / 46(17)$ \\
\hline Twins & $18 / 46(39)$ \\
Maternal fever & $6 / 46(13)$ \\
\hline PPROM & $28 / 46(61)$ \\
Prenatal steroids & $21 / 46(46)$ \\
\hline IUGR & $8 / 46(17)$ \\
Vaginal delivery & $14 / 46(30)$
\end{tabular}

PPROM, preterm premature rupture of membranes; IUGR, intrauterine growth restriction.

Table 3. Neonatal factors.

\begin{tabular}{lc} 
Male & N $(\%)$ \\
SGA & $17 / 46(37)$ \\
BPD & $10 / 46(21)$ \\
Pneumothorax & $29 / 46(63)$ \\
\hline Malformation & $7 / 46(15)$ \\
PDA any treatment & $3 / 46(6)$ \\
\hline ROP any treatment & $31 / 46(67)$ \\
Abdominal surgery & $14 / 46(30)$ \\
\hline Normal/minor brain MRI abnormalities & $14 / 46(30)$ \\
\hline
\end{tabular}

SGA, small for gestational age; BPD, bronchopulmonary dysplasia; PDA, patent ductus arteriosus; ROP, rethinopathy of prematurity; MRI, magnetic resonance imaging.

\begin{tabular}{|c|c|c|c|c|c|}
\hline \multirow[b]{2}{*}{$G A$} & \multicolumn{5}{|c|}{ NFS at 2 years } \\
\hline & 0 & 1 & 2 & 3 & 4 \\
\hline 23 & & & & $\bullet \bullet$ & $\bullet \bullet$ \\
\hline 24 & - & $\bullet$ & $\bullet \bullet \bullet \bullet \bullet \bullet$ & $\bullet \bullet \bullet$ & $\bullet \bullet$ \\
\hline 25 & $\bullet \bullet \bullet$ & $\bullet \bullet \bullet \bullet \bullet \bullet \bullet \bullet$ & 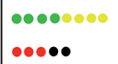 & $\bullet \bullet$ & \\
\hline
\end{tabular}

Figure 1. Neurofunctional score and Griffith's general quotient at 2 years of corrected age according to gestational age. Each infant is represented by a dot. Green dots represent babies with Griffith's general quotient $\geq 88$, yellow dots a general quotient between 76 and 87 , red dots a general quotient $<76$, black dots stand for Griffith's test not performed. 
At the analysis of variance PPROM, absence of prenatal steroids, IUGR, male, lower GA, and severely abnormal MRI were significantly associated with higher NFS score (Table 4) and lower mean Griffith's GQ (Table 5) at 2 years CA.

At the multivariate analysis gestational age, gender and the presence of major brain abnormalities remained significantly associated with severely impaired NFS while no independent factor was identified for the Griffith's GQ (Tables 6 and 7).

\section{Discussion}

Our results strongly support the evidence that survival as well as long-term neurobehavioral outcomes in extremely premature infants are significantly related to gestational age at birth: the lower the gestational age, the higher the risk for death or adverse outcome.

The survival rates observed in our population of infants are similar to those reported by larger studies as the EPICure study ${ }^{4}$ and almost

Table 4. Effect of prenatal and perinatal factors on outcome at 2 years. P values of one-way ANOVA.

\begin{tabular}{lcc} 
Assisted reproduction & $\begin{array}{c}\text { Griffith's GQ } \\
\text { (P value) }\end{array}$ & $\begin{array}{c}\text { NFS score } \\
\text { (P value) }\end{array}$ \\
Twins & 0.979 & 0.543 \\
\hline Gestational hypertension & 0.512 & 0.535 \\
Maternal fever & 0.409 & 0.316 \\
\hline PPROM & 0.676 & 0.457 \\
Prenatal steroids & 0.388 & $0.033^{*}$ \\
IUGR & $0.013^{*}$ & $0.011^{*}$ \\
Vaginal delivery & $0.010^{*}$ & $0.035^{*}$ \\
\hline
\end{tabular}

GQ, general quotient; NFS, neurofunctional scale; PPROM, preterm premature rupture of membranes; IUGR, intrauterine growth restriction. ${ }^{*} \mathrm{P}<0.05$.

Table 5. Effect of neonatal factors on outcome at 2 years. $P$ values of one-way ANOVA.

\begin{tabular}{|c|c|c|}
\hline & $\begin{array}{l}\text { Griffith's GQ } \\
\text { (P value) }\end{array}$ & $\begin{array}{l}\text { NFS score } \\
\text { (P value) }\end{array}$ \\
\hline Male & 0.01 & $<0.01$ \\
\hline SGA & 0.45 & 0.36 \\
\hline $\mathrm{GA}$ & $<0.01$ & $<0.01$ \\
\hline Neonatal weight & 0.36 & 0.85 \\
\hline $\mathrm{HC}<10^{\circ} \mathrm{pcl}$ & 0.12 & 0.68 \\
\hline $\mathrm{BPD}$ & 0.07 & 0.31 \\
\hline Pneumothorax & 0.06 & 0.12 \\
\hline Sepsis & 0.99 & 0.93 \\
\hline PDA any treatment & 0.95 & 0.7 \\
\hline ROP any treatment & 0.06 & 0.02 \\
\hline Abdominal surgery & 0.49 & 0.5 \\
\hline Severely abnormal brain MRI & $<0.01$ & $<0.01$ \\
\hline
\end{tabular}

double for each additional gestational week from 23 to 25 weeks. Despite the advances in perinatal care, we did not observe any significant improvement over an 8-year period, when two different study periods were considered.

Neurobehavioral development appears to be mainly related to gestational age with the most unfavorable outcome occuring in babies born at 23 weeks' gestation, at the limit of viability. Griffith's GQ was $\geq 76$ in $62 \%$ and $\geq 88$ in $33 \%$ of babies born between 24 and 25 weeks. No severe motor disabilities were found in $81 \%$ of babies born between 24 and 25 weeks.

The poor outcome of babies born at 23 weeks of gestational age was probably related to the small number of babies, all with unfavorable conditions at birth (male gender and inadequate prenatal steroid prophylaxis). Caution is needed when comparing results from different studies on long-term outcome in survivors of extremely preterm birth as differences in the neonatal management at birth (proactive approach) profoundly affect survival and rate of disability.

The occurrence of focal brain lesions plays a pivotal role in determining child neurodevelopment. Advances in MRI techniques have allowed an accurate assessment of brain development and gestational age is confirmed to be the main determinant of brain vulnerability to different types of brain lesions. Brain vulnerability is therefore age-dependent and the concept of gestationally determined brain vulnerability has been recently emphasized: the site and nature of the injury sustained being determined by a combination of the characteristics of the insult, the specific tissue and cell vulnerability and the gestation of the infant at birth. ${ }^{19}$

However, even in the absence of overt brain lesions, several perinatal and postnatal factors have been demonstrated to affect the microstructure of the developing white and grey matter with a potential impact on long-term neurodevelopmental outcome.

In our study IUGR, unlike SGA, was associated with an increased risk for adverse neurobehavioral outcome although it was not significant at the multivariate analysis. Intrauterine growth restriction, due to pla-

Table 6. Multivariate analysis for neurofunctional scale at 2 years.

\begin{tabular}{|c|c|c|}
\hline & Variables & P value \\
\hline \multirow{7}{*}{ NFS score } & IUGR & 0.14 \\
\hline & $\mathrm{GA}$ & $<0.01$ \\
\hline & MRI & $<0.01$ \\
\hline & PPROM & 0.05 \\
\hline & Prenatal steroids & 0.06 \\
\hline & Male & $<0.01$ \\
\hline & ROP any therapy & 0.19 \\
\hline
\end{tabular}

NFS, neurofunctional scale; IUGR, intrauterine growth restriction; GA, gestational age; MRI, magnetic resonance imaging; PPROM, preterm premature rupture of membranes; ROP, rethinopathy of prematurity.

Table 7. Multivariate analysis for Griffith's general quotient at 2 years.

\begin{tabular}{ccc} 
GQ & Variables & P value \\
\cline { 2 - 3 } & Male & 0.42 \\
\cline { 2 - 2 } IUGR & 0.3 \\
\hline Prenatal steroids & 0.06 \\
\hline MRI & 0.13 \\
\hline GA & 0.08 \\
\hline
\end{tabular}

$\overline{\mathrm{GQ}}$, general quotient; IUGR, intrauterine growth restriction; GA, gestational age; MRI, magnetic resonance imaging; PPROM, preterm premature rupture of membranes; ROP, rethinopathy of prematurity. 
cental insufficiency leading to reductions in placental nutrient transfer, hypoxia, and progressive metabolic deterioration in almost any fetal organ system, has been associated with increased risk of perinatal mortality and morbidity. ${ }^{20-23}$ Several follow-up studies have described neurological impairments and worse neurodevelopmental outcomes in children born with IUGR. ${ }^{21,24}$ However, long-term neurological impairment frequently seen in IUGR infants cannot be attributed to the presence of overt brain lesions. The risk of brain damage in IUGR neonates could be related to both intrauterine compromised and premature birth. We recently demonstrated that, although gestational age at birth is the predominant factor affecting cerebral maturation, IUGR babies with brain sparing in utero have a mild delay in brain myelination. We speculated that in our study the lack of statistical association between IUGR and worse outcome at the multivariate analysis might be related to the small number of babies. 11

PPROM has been reported to be risk factor for unfavorable outcome, probably due to the underlying physiopathological mechanisms related to chorioamnionitis; ${ }^{25,26}$ however, in this study we had no data on placental histology.

\section{Conclusions}

Although this study has several limitations - mainly related to the small sample size over a quite long period of time - these findings are relevant for clinicians counselling families facing an unavoidable extremely preterm birth.

\section{References}

1. Field D, Draper ES, Fenton A, et al. Rates of very preterm birth in Europe and neonatal mortality rates. Arch Dis Child-Fetal 2009;94:253-6.

2. Moore T, Hennessy EM, Myles J, et al. Neurological and developmental outcome in extremely preterm children born in England in 1995 and 2006: the EPICure studies. Brit Med J 2012;345:e7961.

3. Leviton A, Fichorova RN, O'Shea TM, et al. Two-hit model of brain damage in the very preterm newborn: small for gestational age and postnatal systemic inflammation. Pediatr Res 2013;73:362-70.

4. Costeloe K, Hennessy E, Haider S, et al. Short term outcomes after extreme preterm birth in England: comparison of two birth cohorts in 1995 and 2006 (EPICure studies). Brit Med J 2012;345:e7976.

5. Allen MC, Aucott S, Cristofalo EA, et al. Extrauterine neuromaturation of low risk preterm infants. Pediatr Res 2009;65:542-7.

6. Volpe JJ. Brain injury in premature infants: a complex amalgam of destructive and developmental disturbances. Lancet Neurol 2009;8:110-24.

7. Rombaldi Bernardi J, de Souza Escobar R, Ferreira CF, Pelufo Silveira P. Fetal and neonatal levels of omega-3: effects on neurodevelopment, nutrition, and growth. Sci World J 2012;2012:202473.

8. Kleiber ML, Mantha K, Stringer RL, Singh SM. Neurodevelopmental alcohol exposure elicits long-term changes to gene expression that alter distinct molecular pathways dependent on timing of exposure. J Neurodev Dis 2013;5:6.
9. Rees S, Harding R, Walker D. The biological basis of injury and neuroprotection in the fetal and neonatal brain. Int $\mathrm{J}$ Dev Neurosci 2011;29:551-63.

10. Walker DM, Marlow N, Upstone L, et al. The growth restriction intervention trial: long-term outcomes in a randomized trial of timing of delivery in fetal growth restriction. Am J Obstet Gynecol 2011;204:34.

11. Ramenghi LA, Martinelli A, De Carli A, et al. Cerebral maturation in IUGR and appropriate for gestational age preterm babies. Reprod Sci 2011;18:469-75.

12. Ball G, Counsell SJ, Anjari M, et al. An optimised tract-based spatial statistics protocol for neonates: applications to prematurity and chronic lung disease. Neuroimage 2010;53:94-102.

13. Bonifacio SL, Glass HC, Chau V, et al. Extreme premature birth is not associated with impaired development of brain microstructure. J Pediatr 2010;157:726-32.

14. Anjari M, Srinivasan L, Allsop JM, et al. Diffusion tensor imaging with tract-based spatial statistics reveals local white matter abnormalities in preterm infants. Neuroimage 2007;35:1021-7.

15. Short EJ, Klein NK, Lewis BA, et al. Cognitive and academic consequences of bronchopulmonary dysplasia and very low birth weight: 8-year-old outcomes. Pediatrics 2003;112:e359.

16. Robertson C. Catch-up growth among very-low-birth-weight preterm infants: a historical perspective. J Pediatr 2003;143:145-6.

17. Giannì ML, Picciolini 0 , Vegni $\mathrm{C}$, et al. Twelve-month neurofunctional assessment and cognitive performance at 36 months of age in extremely low birth weight infants. Pediatrics 2007;120:1012-9.

18. Griffiths R. The abilities of young children. London, UK: The Test Agency; 1984.

19. Ramenghi LA, Fumagalli M, Supramaniam V. Brain development and perinatal vulnerability to cerebral damage. In: Buonocore G, Bracci R, Weindling M, eds. Neonatology. A practical approach to neonatal management. Amsterdam, The Netherlands: Springer Verlag; 2012. pp. 1067-78.

20. Baschat AA, Cosmi E, Bilardo CM, et al. Predictors of neonatal outcome in early-onset placental dysfunction. Obstet Gynecol 2007;109:253-61.

21. Leitner Y, Fattal-Valevski A, Geva R, et al. Neurodevelopmental outcome of children with intrauterine growth retardation: a longitudinal, 10-year prospective study. J Child Neurol 2007;22:580-7.

22. Garite TJ, Clark R, Thorp JA. Intrauterine growth restriction increases morbidity and mortality among premature neonates. Am J Obstet Gynecol 2004;191:481-7.

23. Bernstein IM, Horbar JD, Badger GJ, et al. Morbidity and mortality among very-low-birth-weight neonates with intrauterine growth restriction. The Vermont 0xford Network. Am J Obstet Gynecol 2000;182:198-206.

24. Kok JH, den Ouden AL, Verloove-Vanhorick SP, Brand R. Outcome of very preterm small for gestational age infants: the first nine years of life. Brit J Obstet Gynaec 1998;105:162-8.

25. Newton ER. Preterm labor, preterm premature rupture of membranes, and chorioamnionitis. Clin Perinatol 2005;32:571-600.

26. Osmanağaoğlu MA, Unal S, Bozkaya H. Chorioamnionitis risk and neonatal outcome in preterm premature rupture of membranes. Arch Gynecol Obstet 2005;271:33-9. 